\title{
PROCRASTINACIÓN ACADÉMICA, VIVENCIAS ACADÉMICAS Y BIENESTAR PSICOLÓGICO EN ESTUDIANTES UNIVERSITARIOS DE PSICOLOGÍA - ICA
}

\author{
Academic procrastination, academic experiences and psychological well-being \\ in psychology university students - Ica
}

Katia Marilyn Chacaltana Hernández*

\begin{abstract}
Resumen
El presente estudio tiene como objetivo determinar la relación entre la procrastinación académica, vivencias académicas y el bienestar psicológico en estudiantes de psicología de Ica, de II, IV, VI y VIII ciclos.

La muestra estuvo conformada por 162 estudiantes, con edades entre los 16 y 37 años. La investigación es de nivel descriptivo, de tipo sustantivo y diseño correlacional - causal. Se utilizaron como instrumentos de recolección de datos: La Escala de Procrastinación Académica de Busko, el Cuestionario de Vivencias Académicas (QVA-R) y la Escala de Bienestar Psicológico de Ryff.

Los resultados demuestran que existe una relación estadísticamente significativa entre la procrastinación académica, las vivencias académicas y el bienestar psicológico. Obteniendo que la adaptación al estudio, la adaptación personal (factores de las vivencias académicas), el crecimiento personal, la madurez personal y la autoaceptación (factores del bienestar psicológico), son factores predictivos de la procrastinación académica.
\end{abstract}

Palabras clave: Procrastinación, vivencias, adaptación, bienestar, universitarios

\begin{abstract}
The present study aims to determine the relationship between academic procrastination, academic experiences and psychological well-being in psychology students from Ica, of II, IV, VI and VIII cycles.

The sample consisted of 162 students, aged between 16 and 37 years. The research is descriptive level, substantive type and correlational - causal design. The following data collection instruments were used: Busko's Academic Procrastination Scale, the Academic Experiences Questionnaire (QVA-R) and the Ryff Psychological Well-being Scale.

The results show that there is a statistically significant relationship between academic procrastination, academic experiences and psychological well-being. Obtaining that adaptation to study, personal adaptation (factors of academic experiences), personal growth, personal maturity and self-acceptance (factors of psychological wellbeing), are predictive factors of academic procrastination.
\end{abstract}

Keywords: Procrastination, experiences, adaptation, welfare, university - students.

* Docente de la Universidad Nacional San Luis Gonzaga, UNICA. katiam_16_6@ hotmail.com, Lima - Perú. ORCID: 0000-0003-0327-6152 


\section{INTRODUCCIÓN}

En la actualidad, la vida universitaria genera nuevos retos que se afrontan día a día, a la par, aumenta el interés por el estudio de esta. Los temas de éxito y deserción que antes se limitaban a la educación primaria y secundaria se trasladaron al ámbito universitario, aumentando el interés por investigar el fenómeno de transición y adaptación universitaria desde que los estudiantes se encuentran en el primer año (Casanova, Araújo y Almeida, 2020).

En los últimos años, investigaciones en el área de la educación superior identifican, en los estudiantes, problemas en el ámbito de la concentración y ansiedad (Barraza y Silerio, 2007, como se citó en Chau y Saravia, 2014), y la dificultad en el manejo del estrés académico (Chau y Tavera, 2012, como se citó en Chau y Saravia, 2014), así como, angustia o desesperación. Por otro lado, se identifica un desgano conductual, como una importante reacción que influiría en el desarrollo de las labores académicas, uno de los datos más preocupantes en nuestra realidad es compartida por el Ministerio de Educación (MINEDU, 2015), el cual identificó que gran parte de los estudiantes abandonan sus estudios universitarios en los primeros años, siendo una de las razones, la falta de conocimiento sobre su vocación.

Así mismo, se destaca que desarrollar una educación integral es el objetivo más importante de la enseñanza en general y en la universidad en especial, el ingresar a ella demanda poseer un conjunto de habilidades y recursos personales para hacer frente a los retos de un nuevo nivel educativo, ya que no solo se trata de valorar si se poseen conocimientos básicos sobre la carrera, sino de valorar el papel que ejercen las aptitudes, los recursos afectivos y psicológicos, los mismos que permitirán dar respuesta a las exigencias de una carrera universitaria. Son muchos los desafíos a los cuales se enfrentará el estudiante, no sólo aspectos de índole académico o institucional, además de ello, exigencias a nivel interpersonal, a nivel familiar, con sus pares y docentes lo que consolidaría su identidad, autonomía y estrategias de afrontamiento efectivas (Richi, Jorge y Dos Santos, 2006, como se citó en Márquez, Ortiz y Rendón, 2009).

En Perú, según el Instituto Nacional de Estadística e Informática - INEI (2015) el 27,3\% (8 227 879), está representado por los jóvenes peruanos que se encuentran cursando estudios universitarios, es más, se sabe que la cobertura se va incrementando año tras año debido también al incremento de oportunidades para acceder a una universidad, sin embargo, este acceso dependería del nivel socioeconómico de los jóvenes.

$\mathrm{Y}$ es que la sociedad actual, se caracteriza por una creciente expansión de acceso a la Educación Superior, especialmente con un mayor ingreso de estudiantes de grupos étnicos minoritarios o grupos socialmente desfavorecidos, lo que ha diversificado enormemente la población estudiantil a nivel universitario (Casanova, Araujo y Almeida, 2020).

Cabe destacar que reportes de investigación evidencian que la salud constituye un probable factor predictor de futuros problemas o fracasos en el ámbito académico, relacionándose tanto a la salud física y mental, e incluso se la vincula con los niveles funcional y de bienestar (Cornelisse-Vermaat, 2006, como se citó en Chau y Saravia, 2014).

Actualmente, se ha comenzado a reconocer las características personales de los jóvenes en el ambiente universitario y se ha identificado su relevancia, que la universidad ha dejado de ser vista como una fuente educativa que promueve exclusivamente el desarrollo del conocimiento teórico y cuya base es el aprendizaje académico, ahora es percibida como una institución que debe promover la salud, y con ello, lograría proteger el bienestar de sus estudiantes (Consorcio de Universidades, 2013).

La presente investigación se enfoca en el ámbito de la universidad, donde los estudiantes constantemente se encuentran en diversas actividades: exámenes parciales, seminarios, exposiciones, entre otros, las que se relacionan directamente con el estrés académico, por lo que deben ser manejadas adecuadamente por ellos. Conocer el nivel de preparación de los estudiantes universitarios, tanto en lo académico como en lo personal, es el eje fundamental del estudio, por lo que estas condiciones estarían estrechamente relacionadas con un futuro éxito de su ejercicio profesional.

El poder intelectual, el interés por la investigación, la estabilidad y la apertura al cambio, son cualidades básicas que debería poseer cualquier futuro psicólogo, las cuales permitirán generar estrategias para 
solucionar problemas humanos en diversas áreas del desempeño profesional. Es por ello que, en los últimos años, las universidades se han preocupado por formar psicólogos competentes, para posicionarlos como profesionales con habilidades, conocimiento, actitudes y valores (Amador-Soriano, Velásquez-Albo y Alarcón-Pérez, 2018)

El presente estudio considera tres variables, en primer lugar, a la procrastinación académica, la cual es definida como la tendencia a postergar una tarea académica la cual puede ser controlada y ejecutada por el estudiante (Tuckman, 1990), es importante resaltar aquellas características que se relacionan con variables psicológicas que influyen en la forma de relacionarse con sus pares, familia y docentes. La procrastinación académica se entiende también como una práctica de regulación emocional problemática relacionada con la mala salud mental, vinculándola con altos niveles de depresión, ansiedad y estrés (Eisenbeck, Carreno y Uclés-Juárez, 2019)

Como segunda variable, se destaca a las vivencias académicas, las cuales son consideradas como la adaptación universitaria, estrechamente relacionada a la mejora de la salud, siendo definida como el ajuste de desafíos necesarios para lograr la identidad del estudiante y a su vez una adecuada adaptación a la universidad. En ese sentido, autores como Almeida, Ferreira y Soares (2001, como se citó en Chau y Saravia, 2014) relacionan la adaptación universitaria del estudiante con la capacidad autónoma, de competencia, así como el bienestar físico y psicológico del universitario.

Como tercera variable se toma al bienestar psicológico, considerándolo como el proceso de desarrollo hacia la perfección, identificando el verdadero potencial individual, reconociendo $\mathrm{y}$ realizando al máximo todos los talentos (Ryff, 1989). Igualmente, Taylor (1991; citado en Chávez, 2015) propone al bienestar psicológico como componente de la salud que se manifiesta en todo ámbito de la actividad humana. Pero el bienestar también ha sido asociado al sentido de la felicidad, por lo que Sánchez (1998, citado en Chávez, 2015) lo define como el grado de evaluación de vida por parte de una persona, que incluye componentes sobre la satisfacción con la vida, no presencia de depresión y poseer emociones positivas.
Al realizar la revisión de estudios publicados en diferentes fuentes de información sobre estas tres variables de estudio, no se han encontrado investigaciones que planteen la misma propuesta, sin embargo, se destacan estudios en los que se presentan por lo menos dos de las tres variables a trabajar.

A nivel nacional, que encuentran relación entre el bienestar psicológico y la procrastinación académica (Hernández, 2016; Yarlequé y otros, 2016) los antecedentes relacionados a estudios.

A nivel internacional, Morales y Chávez (2017) encontraron que, a mayor adaptación universitaria menor es la procrastinación académica, siendo los estudiantes de ciclos superiores los que cuentan con mayor adaptación universitaria.

Teniendo en cuenta estos resultados, y la base teórica, este estudio ha tenido como principal objetivo determinar la relación entre la procrastinación académica, las vivencias académicas y el bienestar psicológico en estudiantes de psicología.

\section{MÉTODO}

\section{Diseño y población de estudio}

La investigación es de nivel descriptivo, de tipo sustantivo y diseño correlacional - causal.

La población del presente estudio la constituyen los 272 estudiantes matriculados durante el año académico 2017-II, de la facultad de Psicología. Para la selección de la muestra se consideró pertinente determinar las características específicas de la misma mediante dos tipos de criterios: 1. Los criterios de inclusión: Estudiantes hombres y mujeres, que pertenezcan a una universidad nacional de Ica, cuyas edades van de los 16 a 37 años y 2. Cuenten con una asistencia regular (70\% de asistencia como mínimo) durante el semestre académico evaluado.

La selección de la muestra se realizó a través de la aplicación del muestreo probabilístico, llevado a cabo con un margen de error del $5 \%$ y a un nivel de confianza del 95\%, según los criterios señalados por Hernández, Fernández y Baptista (2014). 
De esta forma, de los 272 estudiantes que conformaron la población, la muestra probabilística es de 162 estudiantes.

\section{Instrumentos de medición}

Para obtener la información requerida, se utilizaron las siguientes pruebas: Escala de procrastinación académica, Cuestionario de vivencias académicas y Escala de bienestar psicológico.

La escala de procrastinación académica fue elaborada por Busko (1998) en la Universidad de Guelph (Canadá), cuenta con evidencias de la validez de la estructura interna, de igual manera es confiable, el coeficiente alfa de Cronbach, es de ,86, con un error de medición de ,123. Fue traducido al castellano por Álvarez en el 2010, contando con validez de la estructura interna y con una medida de adecuación de muestreo de Kaiser - Meyer - Oklin de ,80. Además, la prueba de esfericidad de Bartlett alcanzó un valor significativo (Chi-cuadrado $=701,95, \mathrm{p}<05)$.

El cuestionario de vivencias académicas fue elaborado por Almeida (1997), en la Universidad de Minho (Portugal). Presenta alfa de Cronbach superior a ,85. En el ámbito nacional, Alva (2015), desarrolló la adaptación del instrumento, utilizando procedimientos técnicos y metodológicos que brindaron como resultado que esta escala cuenta con evidencias de validez de su constructo. Respecto a la confiabilidad, las dimensiones alcanzaron valores alfa de Cronbach entre, 71 hasta, 80 .

La escala de bienestar psicológico fue elaborada por Ryff (1989) en Wisconsin. Se halla consistencia interna del instrumento encontrando coeficientes alfa de Cronbach que iban de ,86 a ,93. En el Perú, Cubas (2003), realizó una traducción y una validación semántica del instrumento. Posee una confiabilidad con un coeficiente alfa de Cronbach de ,89 para la escala total y entre ,78 y ,87 para las subescalas. Cubas realizó también el análisis ítem-test, cuyos valores fueron superiores a ,50, lo que implica una estructura jerárquica a modo de pirámide que explica el 64,1\% de la varianza.

\section{Análisis estadístico}

Para el análisis de los datos en primer lugar, se utilizaron estadísticos descriptivos como: la media, la desviación estándar, mediana, entre otros. Además, para el análisis de los datos complementarios en el que se trata de establecer la existencia o no de diferencias significativas, se optó por aplicar la prueba U de Mann Whitney. Finalmente, se realizó el análisis de regresión lineal para identificar la predictividad entre las variables.

\section{Consideraciones éticas}

Todos los participantes de la investigación fueron informados de los objetivos del estudio, mediante el consentimiento informado por escrito.

\section{RESULTADOS}

El propósito principal de la investigación es determinar la relación entre la procrastinación académica, las vivencias académicas y el bienestar psicológico de estudiantes de Psicología.

Para ello se contó con la participación de 162 estudiantes universitarios, la mayoría de ellas fueron mujeres (83\%). La muestra en su conjunto fue joven, ya que la edad promedio era 20.3 años $(\mathrm{DE}=2.863)$. Así mismo, sólo una pequeña parte de la muestra (21\%) además de estudiar, también trabaja.

Para realizar la contrastación de la hipótesis, la cual plantea que algunos factores del bienestar psicológico y de las vivencias académicas son predictores de la procrastinación académica en estudiantes de Psicología, se llevó a cabo el análisis de regresión lineal múltiple a través del método de pasos sucesivos, método que analiza los grados de significación de los modelos, eliminando aquellos que no son adecuados.

El análisis de regresión lineal múltiple busca identificar el rol predictor de cada una de las variables independientes sobre la procrastinación académica (variable dependiente), en tal sentido, el mejor modelo identifica las siguientes variables: adaptación al estudio, adaptación personal (dimensiones de vivencias académicas), crecimiento personal, madurez personal y baja autoaceptación personal (del bienestar psicológico); como aquellas que logran ser predictores de la procrastinación académica.

En la tabla 1 se presentan los valores del análisis de varianza de la regresión lineal múltiple, la cual es muy significativa $(\mathrm{F}=11,903, \mathrm{p}<, 001)$; así mismo, se 
observa una correlación múltiple moderada y positiva $(\mathrm{R}=, 664)$ siendo el coeficiente de determinación (R2) igual a ,441, lo cual indica que el $44,1 \%$ de la variación de la procrastinación académica está explicada por la adaptación al estudio, la adaptación personal, el crecimiento personal, la madurez personal y la baja autoaceptación personal. Siendo las primeras dos, correspondientes a la variable de vivencias académicas, y las tres restantes pertenecen a la variable de bienestar psicológico.

\section{Tabla 1}

Análisis de varianza de un factor del análisis de regresión múltiple.

\begin{tabular}{lcrccc}
\hline Modelo & $\begin{array}{l}\text { Suma de } \\
\text { cuadrados }\end{array}$ & gl & $\begin{array}{c}\text { Media } \\
\text { cuadrática }\end{array}$ & F & Sig. \\
\hline Regresión & 3114,015 & 10 & 311,401 & 11,903 & $<, 0001$ \\
Residual & 3950,331 & 151 & 26,161 & & \\
Total & 7064,346 & 161 & & & \\
\hline
\end{tabular}

En la tabla 2 se presenta el análisis del rol predictor de cada variable independiente sobre la procrastinación académica, observándose influencias significativas en cada una de ellas, siendo la adaptación al estudio (adaptación universitaria) (Beta $=-, 441$ ) aquella variable con una predicción más fuerte de la procrastinación académica, el valor del coeficiente Beta es negativo, lo cual indica que a mayores dificultades en la adaptación al estudio, la puntuación de la procrastinación académica es más alta.

\section{Tabla 2}

Coeficientes de la ecuación del análisis de regresión múltiple

\begin{tabular}{lcccc}
\hline Modelo & $\begin{array}{c}\text { Coeficientes } \\
\text { no estandarizados } \\
\end{array}$ & $\begin{array}{c}\text { Coeficientes } \\
\text { B }\end{array}$ & $\begin{array}{c}\text { T } \\
\text { Betandarizados } \\
\text { Beta }\end{array}$ & Sig \\
\hline Adaptación personal &, 148 &, 227 & 2,509 &, 013 \\
Adaptación al estudio &,- 495 &,- 441 & $-4,846$ &, 000 \\
Crecimiento personal &,- 168 &,- 325 & $-2,890$ &, 004 \\
Madurez personal &, 201 &, 201 & 2,489 &, 014 \\
$\begin{array}{l}\text { Baja autoaceptación } \\
\text { personal }\end{array}$ &, 057 &, 188 & 1,801 &, 074 \\
\hline
\end{tabular}

En la tabla 3, se encuentra una relación estadísticamente significativa moderada y negativa ( $\mathrm{r}=$ -,446; $\mathrm{p}<, 0001)$ entre la procrastinación académica y las vivencias académicas en estudiantes de Psicología, esto significa que, a mayores vivencias académicas, menor será la procrastinación académica.

Tabla 3

Relación entre la procrastinación académica total y las vivencias académicas

\begin{tabular}{lcc}
\hline \multirow{2}{*}{ Variables } & \multicolumn{2}{c}{ Vivencias Académicas } \\
& $\mathrm{r}$ & $\mathrm{Sig}$. \\
\hline Procrastinación Académica &,- 446 & $<, 0001$ \\
\hline
\end{tabular}

Así mismo, se observa en la tabla 4 que sí existe una relación estadísticamente significativa entre los factores del bienestar psicológico y la procrastinación académica total, entre las que se destacan mayor relación entre la procrastinación y dos de los factores del bienestar: crecimiento personal y baja autoaceptación personal.

Tabla 4

Relación entre los componentes del bienestar psicológico y la procrastinación académica

\begin{tabular}{lcc}
\hline Factores del Bienestar Psicológico & \multicolumn{2}{c}{ Procrastinación Académica } \\
& $\mathrm{r}$ & Sig. \\
\hline Baja autoaceptación personal &, 375 &, 074 \\
Crecimiento personal &,- 508 &, 004 \\
Madurez personal &,- 128 &, 014 \\
Dificultad al establecer relaciones positivas &, 115 &, 682 \\
Concepción del presente &, 154 &, 792
\end{tabular}

En la tabla 5, se observa relación estadísticamente significativa entre los factores del bienestar psicológico y las vivencias académicas. 


\section{Tabla 5}

Relación entre los componentes del bienestar psicológico y las vivencias académicas

\begin{tabular}{lcc}
\hline Factores del Bienestar Psicológico & \multicolumn{2}{c}{ Vivencias } \\
& Académicas & Sig. \\
\hline Baja autoaceptación personal &,- 687 & $<, 0001$ \\
Crecimiento personal &, 712 & $<, 0001$ \\
Madurez personal &, 418 & $<, 0001$ \\
Dificultad al establecer relaciones positivas &,- 608 & $<, 0001$ \\
Concepción del presente &,- 107 &, 175 \\
\hline
\end{tabular}

Finalmente, se realizó un análisis complementario de la presente investigación con respecto a la procrastinación académica, adaptación universitaria y bienestar psicológico, en la cual, no se encuentran evidencias estadísticanente significativas de diferencia entre sexo $(p=, 737)$, ciclo académico $(p=, 373)$ y ocupación $(\mathrm{p}=, 576)$.

\section{DISCUSIÓN}

El propósito principal de este estudio es determinar la relación entre la procrastinación académica, vivencias académicas y el bienestar psicológico de los estudiantes de Psicología, e identificar predictores sobre la procrastinación académica. Al realizar comparaciones entre los resultados de este estudio con otros, se obtuvo resultados similares, es decir, se establece la convergencia y divergencia entre los resultados hallados y el trabajo de otros autores.

Los resultados obtenidos confirmaron la relación significativa entre la procrastinación académica, vivencias académicas y el bienestar psicológico. Así mismo, cabe resaltar que los factores del bienestar psicológico (crecimiento personal, madurez personal y baja autoaceptación personal) y de vivencias académicas (adaptación al estudio y adaptación personal) son predictores de la procrastinación académica encontrando que sí existe relación estadísticamente significativa.

Esto quiere decir que dichas dimensiones: crecimiento personal, madurez personal, baja autoaceptación personal, adaptación al estudio y adaptación personal, en un bajo nivel o inadecuado funcionamiento en los estudiantes universitarios desencadenaría la procrastinación académica, ya que al ser analizados de manera constante, esta podría ser controlada y reducirse el nivel de procrastinación académica, considerando sus factores, como el déficit de autorregulación académica y postergación de actividades.

Estos resultados coinciden, en parte, con el estudio a nivel internacional de Morales y Chávez (2017), los cuales señalaron que, al identificarse un déficit en las vivencias académicas, éste desencadenaría una procrastinación académica en los universitarios.

Es indudable que la vida universitaria le plantea al joven nuevos retos y exigencias que solo las pueden superar si existe un verdadero compromiso. Para perdurar durante todos sus ciclos académicos, el estudiante universitario necesita de una regulación académica personal, expresada a través de la capacidad para organizar sus materiales a tiempo, regulando sus cogniciones, motivaciones y comportamiento de cara a alcanzar sus objetivos académicos (Busko, 1998); por tanto, esto difícilmente se daría si no existe una real adaptación a sus vivencias académicas, lo que implica modificar su estilo de vida donde se promueva una mayor autonomía del estudiante.

El procrastinar, indicaría un déficit de autorregulación, considerando a la persona procrastinadora como aquella que posterga dichas actividades que lo ayudarían a cumplir una meta final (Tuckman, 2005), pues encontrando dificultad en la adaptación universitaria es muy probable que se halle la procrastinación académica.

Ahora bien, se encuentra una relación estadísticamente significativa entre la procrastinación académica y las vivencias académicas. Este hallazgo podría relacionarse con el estudio de Chan en Lima (2011) el cual encuentra influencia directa entre la procrastinación académica y el rendimiento académico de los universitarios, considerando al bajo rendimiento académico, como consecuencia de una inadecuada adaptación universitaria.

En España, Eisenbeck, Carreno y UclésJuárez (2019) manifiestan que cuando el estudiante universitario presenta niveles elevados de procrastinación académica, mayor será su angustia, y ambos a su vez se relacionarán directamente con la 
inflexibilidad psicológica. De igual modo, en estados Unidos, Gustavson y Miyake (2017), demuestran en su estudio actual que los niveles de procrastinación académica están ligados al logro de metas académicas de los estudiantes.

Por otra parte, Cardona (2015) identificó una relación significativa entre la procrastinación y el estrés académico.

Considerando que los autores anteriores no han tomado la variable de vivencias académicas, es importante resaltar, que se ha considerado a las otras variables como aquellas exigencias que están inmersas en la adaptación universitaria (Almeida, Soares y Ferreira, 2000, citados en Chau y Saravia, 2014) como lo son el rendimiento académico, angustia, metas académicas y el estrés académico, tareas estrechamente ligadas, es por ello que se habla de una concordancia en sus resultados.

Tal cual Milgram (2014, citado en Hernández 2016) expone que los componentes emocionales (miedo al fracaso, el miedo al éxito o a lograr menos de lo que se quiere, sentimientos de culpa o ansiedad) están relacionados a la acción de procrastinar, es decir, la combinación de pensamientos, emociones y acciones que toma frente a una responsabilidad, influye en la persona frente a alguna actividad relacionada directamente con sus estudios universitarios. Estos hallazgos concuerdan con los encontrados por Hernández (2016), quien encontró una relación estadísticamente significativa entre la procrastinación académica y sus motivos de procrastinación, con el bienestarpsicológico.Asímismo, concuerdacon García (2014), quien identificó relaciones estadísticamente significativas entre bienestar psicológico, inteligencia emocional, disposición a fluir y procrastinación académica en estudiantes universitarios.

Además, se destaca la concordancia de resultados con la investigación realizada por Yarlequé y otros (2016) en estudiantes de Lima y Junín, quienes manifiestan que los estudiantes que poseen mayor bienestar psicológico evidencian menor procrastinación.

De igual manera, se destaca que si el estudiante no se acepta tal como es, éste no tendrá una adecuada adaptación universitaria o desarrollo de vivencias académicas; similar situación ocurre con la dificultad al establecer relaciones positivas, ya que, si el estudiante se encuentra en la necesidad de comseguir y mantener relaciones positivas, afectaría a sus vivencias académicas.

Estos resultados, sin duda, destacan la importancia del bienestar psicológico (reflejado en sus factores) y su relación significativa con las vivencias académicas o la adaptación universitaria, lo que coincide con el estudio realizado por Chau y Saravia (2014), quienes destacaron que la salud mental y el bienestar de los estudiantes universitarios se ve reflejado mediante comportamientos saludables y estos a su vez influenciarían en una adecuada adaptación universitaria. Además, se concuerda con la investigación realizada por Freire (2014), quien manifiesta que a medida que el estudiante tenga un adecuado bienestar psicológico, logrará un afrontamiento eficaz ante el estrés académico, considerando al estrés académico como un factor inmerso en la adaptación académica, los mismos resultados fueron obtenidos por García (2015). Y, en el caso de Correa, Cuevas y Villaseñor (2016) también encontraron una relación altamente significativa entre el bienestar psicológico y el rendimiento académico de los estudiantes universitarios.

Además, se destaca la relación de la madurez emocional (factor del bienestar psicológico) respecto a las vivencias académicas.

En cuanto a la procrastinación académica, vivencias académicas y bienestar psicológico; no se han hallado diferencias significativas según sexo, ocupación o ciclo académico.

\section{CONCLUSIONES}

- Se encuentra relación significativa entre la procrastinación académica, vivencias académicas y bienestar psicológico.

- Se identifica que la adaptación al estudio, la adaptación personal (factores de las vivencias académicas), el crecimiento personal, la madurez personal y la autoaceptación (factores del bienestar psicológico), son factores predictores de la procrastinación académica. 
- Se recomienda realizar un estudio con las variables: procrastinación académica, vivencias académicas y bienestar psicológico, como réplica en diversas carreras a nivel local, regional y nacional.

- Finalmente, en base a la coyuntura actual que atraviesa el mundo en general, debido a la pandemia del Covid - 19, se recomienda desarrollar investigaciones que tengan como objetivo analizar la influencia de la pandemia, el confinamiento y las clases virtuales en las tres variables propuestas del estudio, las cuales son: procrastinación académica, vivencias académicas y bienestar psicológico.

\section{REFERENCIAS}

Amador-Soriano, K., Velásquez-Albo, M. y AlarcónPerez, L. (2018). Las competencias profesionales del psicólogo desde una perspectiva integral. Revista de educación y desarrollo, 45, 5 - 14. Recuperado de: http://www.cucs.udg.mx/revistas/ edu_desarrollo/anteriores/45/45_Amador.pdf

Busko, D. (1998). Causes and consequences of perfectionism and procrastination: A structural equation model. The University of Guelph, Ontario, Canadá.

Casanova, J., Araújo,A. \& Almeida,L. (2020). Dificuldades na adaptação académica dos estudantes do $1^{\circ}$ ano do Ensino Superior. Revista E-Psi, 9(1), 165-181.Recuperado de: https:// www.researchgate.net/publication/340926335

Chau, C. y Saravia, J. C. (2014). Adaptación universitaria y su relación con la salud percibida en una muestra de jóvenes de Perú. Revista Colombiana de Psicología, 23(2), 269-284. doi: 10.15446/rcp.v23n2.41106

Chavez, C. (2015). Calidad de Vida y bienestar psicológico en personas con discapacidad motriz. de un centro de integración de discapacitados de Trujillo. (Tesis de licenciatura). Universidad Privada Antenor Orrego, Trujillo, Perú.

Consorcio de Universidades. (2013). Guía para universidades saludables. Lima: Consorcio de Universidades.
Eisenbeck, N., Carreno, D. \& Úcles-Juárez, R. (2019). From psychological distress to academic procrastination: Exploring the role of psychological inflexibility. Journal of contextual behavioral science, 13,103-108. Recuperado de: https://doi.org/10.1016/j.jcbs.2019.07.007

Freire, C. (2014). El Bienestar Psicológico en los estudiantes universitarios: Operativización del constructo y análisis de su relación con las estrategias de afrontamiento (Tesis de doctorado). Universidade da Coruña, La Coruña, España.

Gustavson, D. \& Miyake, A. (2017). Academic procrastination and goal accomplishment: A combined experimental and individual differences investigation. Learning and individual differences, 54, 160-172. Recuperado de: http:// dx.doi.org/10.1016/j.lindif.2017.01.010

Hernández, G. (2016). Procrastinación académica, motivos de procrastinacióny bienestar psicológico en alumnos de ingeniería industrial de una universidad de Trujillo (Tesis de licenciatura). Universidad Privada del Norte, La Libertad, Perú.

Instituto Nacional de Estadística e Informática - INEI (2015) PerúSíntesis Estadística 2015. Recuperado el 12 de septiembre de https://www.inei.gob.pe/ media/MenuRecursivo/publicaciones_digitales/ Est/Lib1292/libro.pdf

Márquez, D., Ortiz, S. y Rendón, M. (2009). Cuestionario de Vivencias Académicas en su versión reducida (QVA-r): un análisis psicométrico. Revista colombiana de psicología, 18 (1), 33 - 52. Recuperado de: https://revistas. unal.edu.co/index.php/psicologia/article/ view/1450

Ministerio de Educación (MINEDU, 2015). Guía de orientación de estudios. Recuperado de www. minedu.gob.pe/DeInteres/Publicaciones/guia_ estudios/

Morales, M. y Chávez, J. (2017). Adaptación a la vida universitaria y procrastinación académica en estudiantes de psicología. Revista electrónica del Desarrollo Humano para la innovación, 4(8). Recuperado de: http://www.cdhis.org.mx/index. $\mathrm{php} / \mathrm{CAGI} /$ article/view/121

Ryff, C. (1989). Happiness is everything or is it? Explorations on the meaning of psychological 
well-being. Journal of Personality and Social Psychology, 57(6), 1069-1081. Recuperado de: https://pdfs.semanticscholar.org/7eb5/1dfece4f3 9df7c5c3aefa1276ae1116473a5.pdf

Tuckman, B.W. (1990). Group versus goal-setting effects on the self-regulated performance of students differing in self-efficacy. Journal of Experimental Education, 58, 291-298. Recuperado de: http://psycnet.apa.org/ record/1991-05916-001
Yarlequé , L., Javier, L., Monroe , J. D., Nuñez, E., Padilla, M., ... y Campos, J. (2016). Procrastinación, estrés y bienestar psicológico en estudiantes de educación superior de Lima y Junín. Horizonte de la Ciencia, 6(10), 173-184. Recuperado de: http://ucsp.edu.pe/investigacion/ psicologia/wp-content/uploads/2017/09/Revpsicol-UCSP-2017-1.pdf

Fecha de recepción: 27 de noviembre 2019

Fecha de aceptación: 27 de diciembre 2019 